\title{
Random-forcing model of the mesoscale oceanic eddies
}

\author{
By PAVEL S. BERLOFF \\ Woods Hole Oceanographic Institution, USA and Department of Applied Mathematics \\ and Theoretical Physics, University of Cambridge, Centre for Mathematical Sciences, \\ Wilberforce Road, Cambridge CB3 0WA, UK \\ pberloff@whoi.edu
}

(Received 20 April 2004 and in revised form 27 October 2004)

The role of mesoscale oceanic eddies in driving large-scale currents is studied in an eddy-resolving midlatitude double-gyre ocean model. The reference solution is decomposed into large-scale and eddy components in a way which is dynamically consistent with a non-eddy-resolving ocean model. That is, the non-eddy-resolving solution driven by this eddy-forcing history, calculated on the basis of this decomposition, correctly approximates the original flow. The main effect of the eddy forcing on the large-scale flow is to enhance the eastward-jet extension of the subtropical western boundary current. This is an anti-diffusive process, which cannot be represented in terms of turbulent diffusion. It is shown that the eddy-forcing history can be approximated as a space-time correlated, random-forcing process in such a way that the non-eddy-resolving solution correctly approximates the reference solution. Thus, the random-forcing model can potentially replace the diffusion model, which is commonly used to parameterize eddy effects on the large-scale currents. The eddy-forcing statistics are treated as spatially inhomogeneous but stationary, and the dynamical roles of space-time correlations and spatial inhomogeneities are systematically explored. The integral correlation time, oscillations of the space correlations, and inhomogeneity of the variance are found to be particularly important for the flow response.

\section{Introduction}

Mesoscale eddy fluxes of momentum and potential vorticity are capable of driving large-scale oceanic currents. In the ocean models, the unresolved fluxes are always accounted for by simple mathematical models, i.e. parameterizations. This paper proposes to represent effects of eddy fluxes in a non-eddy-resolving ocean model in terms of random forcing. In the introduction, some background is discussed and the problem is posed. Section 2 describes ocean models used and the diagnostics method, and $\S 3$ analyses statistics of the eddy forcing; $\S 4$ deals with modelling random forcing, and summary and discussion of the results follow in $\S 5$.

\subsection{Background}

The idea of modelling eddy effects by random processes is both attractive and underdeveloped. Some encouraging results have been obtained for simple differential equations (Majda, Timofeyer \& Vanden-Eijnden 1999, 2002, 2003) and advanced toward a realistic barotropic model of the atmosphere (Franzke, Majda \& VandenEijnden 2004). In a broad class of fluid-dynamic models of the homogeneous and isotropic three-dimensional turbulence, the nonlinear interactions (i.e. eddy fluxes) are replaced by random forcing acting in the wavenumber spectral space (e.g. Herring \& 
Kraichnan 1971; Laval, Dubrulle \& Nazarenko 2003). The major weakness of these models is that the random forcing is not constrained by theories, and spatial inhomogeneities and anisotropy are not accounted for. In some geophysical situations, the nonlinear interactions can be replaced completely by random forcing driving linear large-scale models (Farrell \& Ioannou 1995; Whitaker \& Sardeshmukh 1998; Zhang \& Held 1999), but in other situations large-scale nonlinearities can be fundamentally important (Williams 1978). The randomly forced nonlinear singleand double-gyre ocean models have been studied in the context of both wind forcing variability and eddy effects (Treguier \& Huax 1987; Griffa \& Castellari 1991; Seidov \& Marushkevich 1992), and it has been argued that, in the free-slip barotropic dynamics, the forcing variability enhances the single-gyre inertial recirculation, but weakens the double-gyre one. Berloff (2004a) systematically studies the randomly forced baroclinic dynamics in a closed basin. Sensitivity to statistical details of random forcing is studied in Sura \& Penland (2002).

Although turbulent diffusion is the most common approach for modelling eddy effects, some other ideas are being developed as well. In particular, under certain assumptions, eddies can be represented by ensembles of particles advected by the large-scale flow (Laval et al. 2000). Effects of the boundary-layer eddies can be modelled by replacing the no-slip boundary condition with a stochastic one (Mariano, Chin \& Ozgokmen 2003). The Lagrangian averaged Navier-Stokes $\alpha$-model is based on the assumption that fluctuations at length scales shorter than the given length scale, $\alpha$, are passively advected by the large-scale flow component (Holm \& Nadiga 2003). Finally, none of the present modelling approaches, including the turbulent diffusion and random-forcing theories, is closed in the sense that parameters of the eddy model are simply related to the resolved large-scale flow.

\subsection{Statement of the problem}

The problem is addressed in the context of an idealized ocean circulation, but the ideas and the method can be applied to other geophysical flows and general turbulence. First, the history of the eddy flux divergence, that is, the eddy forcing, is diagnosed from the reference eddy-resolving solution of the ocean circulation. The diagnostics are based on a dynamically consistent decomposition of the flow into large-scale and eddy components. This implies that solution of a non-eddy-resolving model driven by the eddy-forcing history correctly approximates the original flow. Since the eddy effects are largely anti-frictional, they cannot be modelled as a diffusion process. The alternative idea is to model the eddy forcing in terms of spatially inhomogeneous space-time correlated random process, and the diagnosed eddy forcing is used as the starting point for determining parameters of the random process. The corresponding non-eddy-resolving model allows us to estimate accuracy and verify assumptions of the random-forcing approach, and to dynamically interpret different statistical properties of the eddies. More specifically, the main questions are the following. What are statistical properties of the diagnosed eddy forcing? What is the appropriate framework for random-forcing models? What are dynamically important properties of the eddy forcing and the corresponding parameters of the random forcing? What is the correct balance between simplicity of the random forcing and quality of the solution?

\section{Ocean model and flow decomposition}

\subsection{Eddy-resolving ocean model}

Here, the eddy-resolving model is used to obtain the reference solution, which contains both the large-scale and eddy components. The model represents the midlatitude 
ocean, with a prescribed density stratification, and in a flat-bottomed square basin with north-south and east-west boundaries. The quasi-geostrophic potential-vorticity equations (Pedlosky 1987) for $N$ dynamically active isopycnal layers are:

$$
\frac{\partial q_{i}}{\partial t}+J\left(\psi_{i}, q_{i}\right)+\beta \frac{\partial \psi_{i}}{\partial x}=\frac{\delta_{i, 1}}{\rho_{1} H_{1}} \nabla \times \tau+\nu \nabla^{4} \psi_{i},
$$

where $\delta_{i, j}=1$ if $i=j ; \delta_{i, j}=0$ if $i \neq j$, and $i$ is the layer index starting from the top; $H_{1}$ and $\rho_{1}$ are the upper-layer depth and density, respectively; and $v$ is the eddy diffusivity coefficient. The meridional planetary vorticity gradient is $\beta$, the surface wind stress is $\tau$, and $J($,) is the Jacobian operator. The potential-vorticity anomalies, $q_{i}$, are connected with the velocity streamfunctions, $\psi_{i}$, through the system of elliptic equations:

$$
\nabla^{2} \psi_{i}-\left(1-\delta_{i, 1}\right) S_{i, 1}\left(\psi_{i}-\psi_{i-1}\right)-\left(1-\delta_{i, N}\right) S_{i, 2}\left(\psi_{i}-\psi_{i+1}\right)=q_{i},
$$

with the stratification parameters $S_{i, 1}$ and $S_{i, 2}$. The horizontal velocity components are found to be

$$
u_{i}=-\frac{\partial \psi_{i}}{\partial y}, \quad v_{i}=\frac{\partial \psi_{i}}{\partial x}
$$

and, on the lateral boundaries, velocity is zero. Also, there is the mass conservation constraint for each layer:

$$
\frac{\partial}{\partial t} \iint \psi_{i}(x, y) \mathrm{d} x \mathrm{~d} y=0 .
$$

The spatial resolution is $7.5 \mathrm{~km}(512 \times 512$ grid points $)$. This grid size is close to the $1 / 12$-degree resolution, at which, it is argued, the mesoscale eddies are marginally resolved (e.g. Bleck et al. 1995). Parameters of the model and basic features of the flow solution are discussed in Appendix A, and the time-mean and instantaneous flow patterns are shown in figures 1 and 2, respectively.

After the initial spin-up from the state of rest, the solution equilibrates statistically. Then, it is computed for $10^{4}$ days and stored for the analysis on the coarsened $129 \times 129$ grid, with 1-day time intervals. This data set is referred to as the coarsegrained (or, projected on the coarse grid), reference eddy-resolving solution, and it has the same spatial resolution as the non-eddy-resolving model. In general, the coarse-graining method can be altered by using a coarse-gridscale filter, but here the simplest option is used, which is equivalent to the common observational practice of estimating pointwise quantities. Also, the filtering would result in underestimating the variance of the flow fluctuations.

\subsection{Eddy forcing and dynamical decomposition algorithm}

The large-scale and eddy components of the flow, arbitrary so far, are denoted with overbar and prime, respectively, and the full flow is:

$$
\boldsymbol{u}(t, \boldsymbol{x})=\overline{\boldsymbol{u}}+\boldsymbol{u}^{\prime}, \quad q(t, \boldsymbol{x})=\bar{q}+q^{\prime} .
$$

The eddy forcing is defined as

$$
f=J(\bar{\psi}, \bar{q})-J(\psi, q)=\nabla \cdot \overline{\boldsymbol{u}} \bar{q}-\nabla \cdot \boldsymbol{u} q=-\nabla \cdot \boldsymbol{F}^{\prime} .
$$

Presumably, the eddy forcing is the eddy effect that is missing from a non-eddyresolving dynamical model of the large-scale flow. There is a caveat here: if the flow decomposition is not consistent with a particular non-eddy-resolving model, then solution of this model forced by the eddy-forcing history will not approximate 
(a)

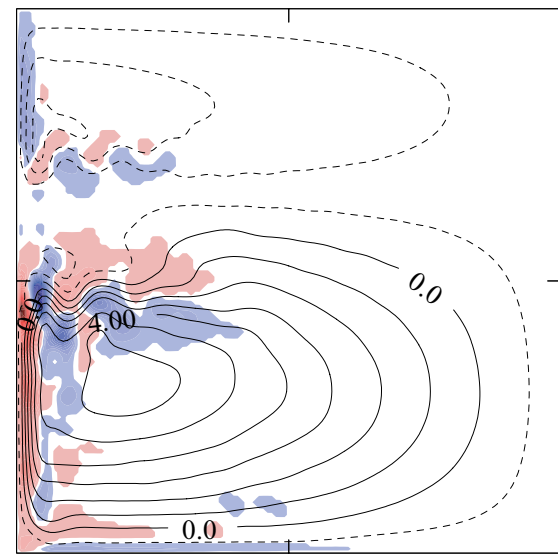

(c)

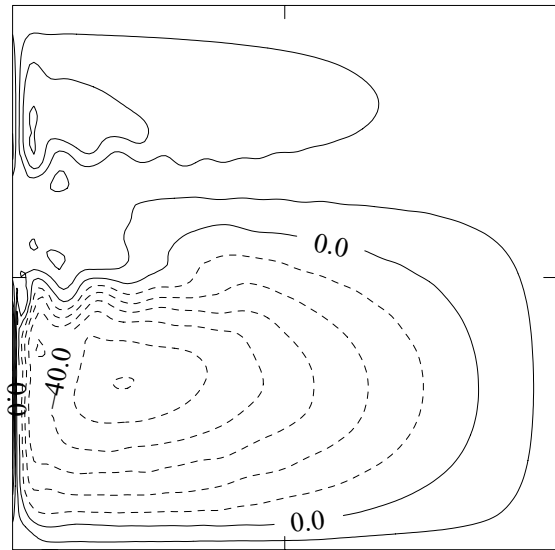

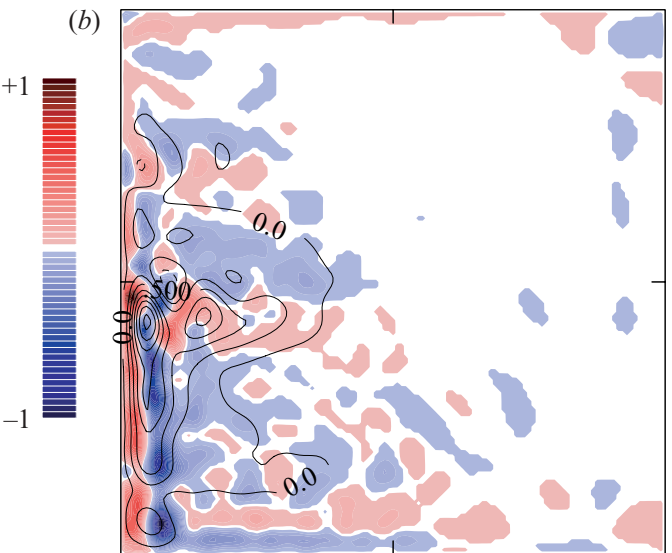

(d)

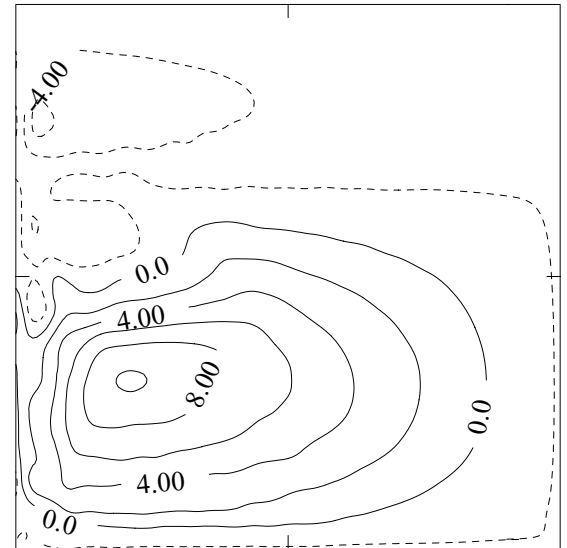

FIGURE 1. The time-mean velocity streamfunction (contours) of the eddy-resolving reference solution and the corresponding time-mean eddy forcing (colour) in the $(a)$ upper and $(b)$ deep ocean. $(c, d)$ The corresponding distributions of the potential vorticity. CIs are $(a) 10^{4} \mathrm{~m}^{2} \mathrm{~s}^{-1}$, (b) $0.25 \times 10^{4} \mathrm{~m}^{2} \mathrm{~s}^{-1},(c) 10 \times 10^{-6} \mathrm{~s}^{-1}$ and $(d) 2 \times 10^{-6} \mathrm{~s}^{-1}$. The negative and positive values of the eddy forcing are normalized by the minimum and maximum values of the eddy forcing over the basin, so that the colour scale varies from -1 to +1 . Here, the minimum and maximum values of the time-mean eddy forcing are: $(a)-39$ and $15.6 \times 10^{-8} \mathrm{~s}^{-2}$ and $(b)-0.96$ and $0.74 \times 10^{-8} \mathrm{~s}^{-2}$.

the original flow. This is because the non-eddy-resolving model will develop its own nonlinear dynamics, which is inconsistent with the large-scale flow evolution implied by (5). If the caveat is overcome, then we can think of forcing the non-eddy-resolving model with random forcing that replaces the real eddy-forcing history - this is a way of parameterizing the eddy effects. On the other hand, if decomposition (5) is done in a purely statistical way, as common in practice, no dynamical consistency is ensured. This is because the corresponding eddy forcing is unrelated to the difference in dynamical degrees of freedom between the full flow and its truncated (i.e. noneddy-resolving) approximation. For example, when a weakly truncated model resolves a large fraction of the eddy population, the corresponding eddy forcing has to be weak.

The dynamical decomposition algorithm is based on integrating and interactively correcting the non-eddy-resolving model solution with the information supplied by the structure of the full flow. This information enters the non-eddy-resolving model 
(a)

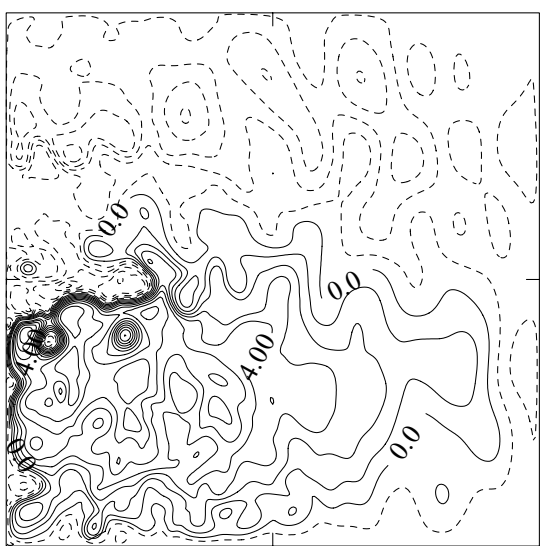

(c)

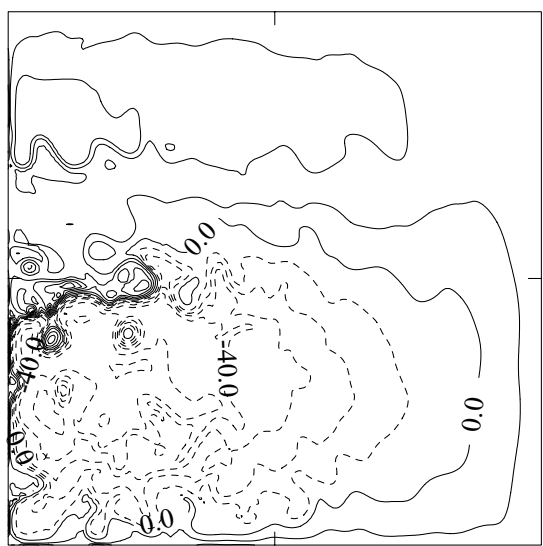

(e)

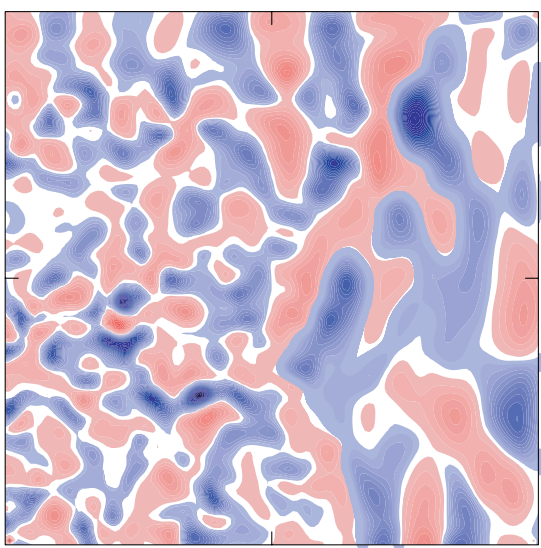

(b)

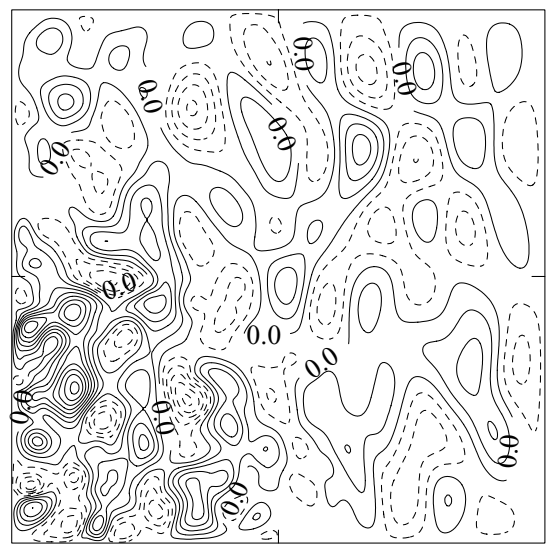

(d)
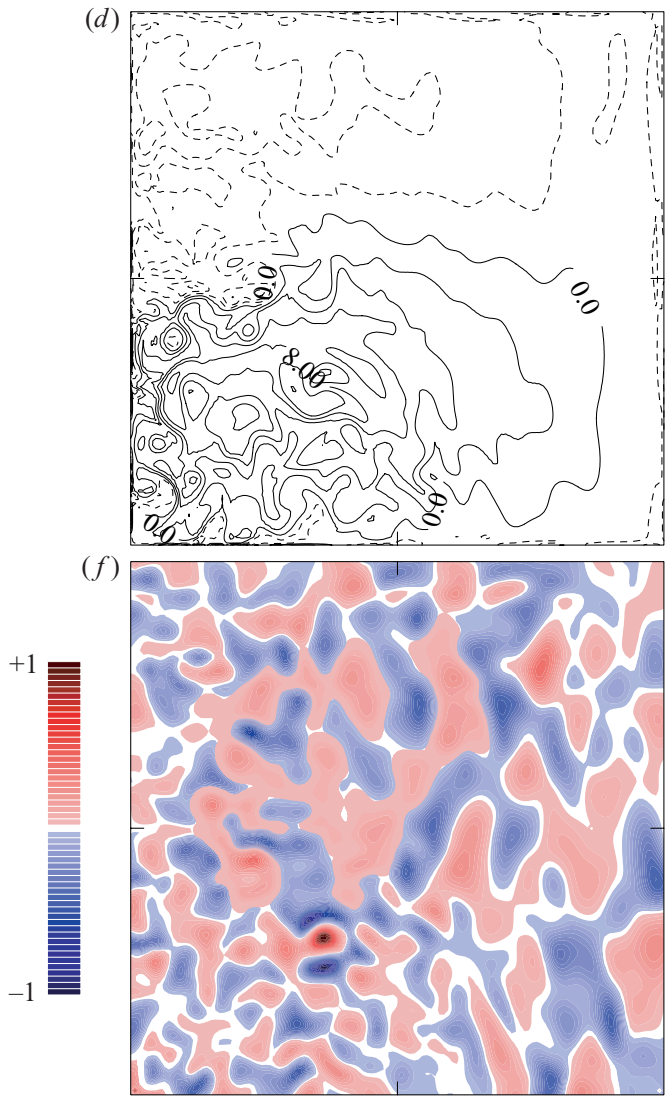

FIGURE 2. Instantaneous velocity streamfunction in the $(a)$ upper and $(b)$ deep ocean. $(c, d)$ The corresponding distributions of the potential vorticity. $(e, f)$ The corresponding patterns of the eddy forcing fluctuation (normalized by $\sqrt{\sigma_{f}}$ ). CIs are $(a) 10^{4} \mathrm{~m}^{2} \mathrm{~s}^{-1},(b) 0.5 \times 10^{4} \mathrm{~m}^{2} \mathrm{~s}^{-1}$, (c) $10 \times 10^{-6} \mathrm{~s}^{-1}$ and $(d) 2 \times 10^{-6} \mathrm{~s}^{-1}$. The negative and positive values of the eddy forcing are normalized by the minimum and maximum values of the eddy forcing over the basin, so that the colour scale varies from -1 to +1 . Here, the minimum and maximum values of the instantaneous eddy forcing are: $(a)-39$ and $15.6 \times 10^{-8} \mathrm{~s}^{-2}$ and $(b)-0.96$ and $0.74 \times 10^{-8} \mathrm{~s}^{-2}$.

in terms of the interactively calculated eddy forcing, and the eddies are continuously and interactively defined in terms of the structural differences between the full and non-eddy-resolving flows. The non-eddy-resolving model is formulated in the same 
way as the eddy-resolving one, except that it has a coarse spatial grid with $30 \mathrm{~km}$ resolution (Berloff 2004b). Also, its eddy diffusivity, $v$, is enhanced by a factor of 10 , in order to parameterize the effects of sub-mesoscale eddy forcing wiped out as a result of the coarse-grid projection of the eddy-resolving solution (Appendix A). Sensitivity study with the enhancement factors of 1 and 100 confirm all of the basic conclusions of the analysis. This is so, because most of the dissipation occurs near the western boundary and the dynamically important eddy fluxes in the eastward-jet region are not very sensitive to the diffusivity enhancement. Given that the coarse grid has four times fewer points in each direction, the grid-scale Reynolds number, $U \Delta / \nu$, where $U$ is some typical velocity and $\Delta$ is the grid scale, is reduced by a factor of 0.4. This is done in order to be able to resolve marginally, by just one point, the Munk scale, $[v / \beta]^{1 / 3}$. In a more advanced application of the ideas developed in this paper, either $\Delta$ or $v$ can be spatially inhomogeneous, so that both the grid-scale Reynolds number is kept fixed and the Munk scale is well resolved.

More specifically, the non-eddy-resolving model is initialized with the initial conditions for the eddy-resolving flow and integrated in time. In each isopycnal layer and at each grid point, the instantaneous eddy forcing is found according to (6), where $J($,$) and \nabla$ are the coarse-grid operators. The large-scale component of the flow, $\bar{\psi}$, is defined as the non-eddy-resolving solution; and the eddy component, $\psi^{\prime}$, is found to be the difference between the full flow and the non-eddy-resolving solution. The non-eddy-resolving model is stepped forward with this eddy-forcing term and the process is repeated for the next time step. Thus, the eddies are literally the unresolved fluctuations of the non-eddy-resolving dynamics, but, nevertheless, they are accounted for in terms of the (residual) eddy forcing.

In the upper ocean, the time-mean eddy forcing has the dipolar structure that enhances the main eastward jet by driving cyclonic and anticyclonic recirculations to the north and south of it, and in the deep ocean it acts in the opposite way (figure 1). The detailed study of the structure of the time-mean eddy fluxes and forcing is in Berloff (2004b). The eddy-forcing history, (6), includes divergences of the largescale/eddy and eddy/eddy potential-vorticity fluxes, and both of them substantially contribute to the time-mean component and variance of the eddy forcing. Importance of the large-scale advection in driving the recirculations by supplying potentialvorticity anomalies is argued in Cessi, Ierley \& Young (1987) and Cessi (1988), and the role of the time-mean eddy/eddy potential-vorticity flux is studied in Jayne, Hogg \& Malanotte-Rizzoli (1996).

\subsection{Relation to other decomposition methods}

Decomposing turbulence by dynamic models, as is done here, is not common. An alternative method, applied to the large-eddy simulation of the isotropic turbulence, calculates the eddy forcing by making use of the truncated approximation of the Navier-Stokes equation solved on a finer grid (Domaradzki, Loh \& Yee 2002). This method has no enhanced eddy diffusivity, therefore the energy gradually piles up at small scales, and to avoid problems, the fine-grid flow has to be periodically reinitialized with the parallel coarse-grid solution. The dynamical decomposition method is simpler and more straightforward, but it is expected to work only when the eddy forcing is a leading-order term. Fortunately, this is the case in the oceanic gyres. Although the method proposed here also involves corrections given by differences between the modelled and reference flows, it is different from the method based on calculating residual forcing from the tendency term (D'Andrea \& Vautard 2000) and from the approach in which the residual forcing is used to correct the eddy diffusivity 
coefficient (Kaas et al. 1999). It is also different from the approach in which the solution is corrected by the time-mean component rather than the full history of the eddy forcing (Marshall \& Molteni 1993). Here, an attempt to account for other eddy effects by correcting the diffusion operator and the $\beta$-term destroys the leadingorder linear balance in the western-boundary viscous layer and corrupts the integral potential-vorticity balance. In turn, this induces solution runaway characterized by exaggerated gyres.

\subsection{Sensitivity to coarse grid}

Finally, sensitivity to the choice of the coarse grid is tested by projecting the eddyresolving solution on the $65 \times 65$ grid and by using the corresponding non-eddyresolving model. Although the corresponding non-eddy-resolving solution produces an enhanced eastward jet, the quality of the solution is worse, mainly because of the excessive broadening of the western boundary currents and their eastward jets, and to the poor resolution of the intense mesoscale eddies, such as those in figure 2. The eddy forcing retains its general statistical features, except for the small-scale structure of spatial correlations. Here, it is argued that although the method works with tolerable quality for very coarse resolutions, the optimal coarse grid should marginally resolve the first baroclinic Rossby deformation radius, so that large transient coherent structures around the eastward jet, such as the meanders and vortices in figure 2, remain substantially represented. The basis for this reasoning is that such coherent structures dominate material transport (e.g. Armenio, Piomelli \& Fiorotto 1999; Berloff \& McWilliams 2002), which has to be modelled properly if the method is advanced to more comprehensive ocean models (see also discussion in $\S 5.3)$.

\section{Statistical properties of the eddy-forcing fluctuations}

This section studies statistical properties of the eddy-forcing fluctuations. The fluctuation statistics are described by the spatial distribution of the space-time statistical moments:

$$
\Xi\left(\boldsymbol{x}_{0}, \boldsymbol{x}, \tau, m, n\right)=\left\langle f^{m}\left(t, \boldsymbol{x}_{0}\right) f^{n}\left(t+\tau, \boldsymbol{x}_{0}+\boldsymbol{x}\right)\right\rangle,
$$

where \langle\rangle indicates the time averaging. Here, the focus is on the second-order covariance function (i.e. $m=n=1$ ), under the hypothesis that it contains all of the important information. The full covariance is separated into the temporal (i.e. autocovariance) and spatial components, and the latter is separated into the horizontal and vertical components. The correlation function is obtained by dividing by the covariance function with square roots of the corresponding variances. All of these quantities yield the autocorrelation, $R_{T}$, horizontal correlation, $R_{H}$, and vertical correlation, $R_{V}$, functions:

$$
\begin{aligned}
R_{T}\left(\tau, \boldsymbol{x}_{0}\right) & =\frac{\left\langle f\left(t, \boldsymbol{x}_{0}\right) f\left(t+\tau, \boldsymbol{x}_{0}\right)\right\rangle}{\sigma_{f}\left(\boldsymbol{x}_{0}\right)}, \\
R_{H}\left(x, y ; \boldsymbol{x}_{0}\right) & =\frac{\left\langle f\left(t, \boldsymbol{x}_{0}\right) f\left(t, x, y, z_{0}\right)\right\rangle}{\sigma_{f}^{1 / 2}\left(\boldsymbol{x}_{0}\right) \sigma_{f}^{1 / 2}\left(x, y, z_{0}\right)}, \\
R_{V}\left(z ; \boldsymbol{x}_{0}\right) & =\frac{\left\langle f\left(t, \boldsymbol{x}_{0}\right) f\left(t, x_{0}, y_{0}, z\right)\right\rangle}{\sigma_{f}^{1 / 2}\left(\boldsymbol{x}_{0}\right) \sigma_{f}^{1 / 2}\left(x_{0}, y_{0}, z\right)},
\end{aligned}
$$



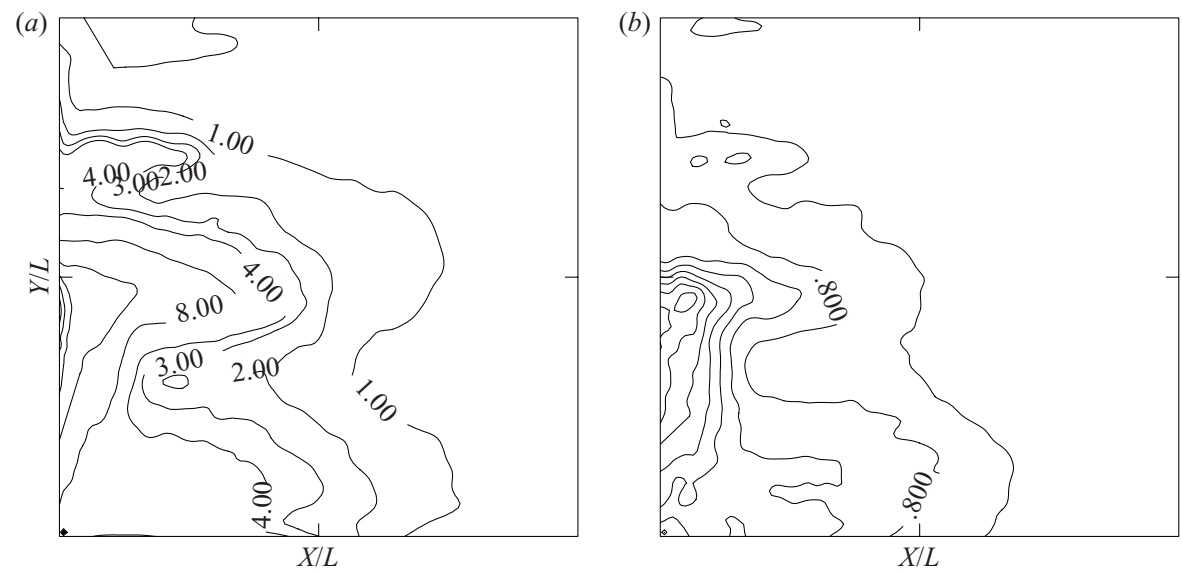

FIgURE 3. Distribution of $\sqrt{\sigma_{f}}$ in the $(a)$ upper and $(b)$ deep ocean. $(a) \mathrm{CI}=10^{-12} \mathrm{~s}^{-2}$ for $\sqrt{\sigma_{f}} \leqslant 4 \times 10^{-12} \mathrm{~s}^{-2}, 20 \times 10^{-12} \mathrm{~s}^{-2}$ for $\sqrt{\sigma_{f}} \geqslant 20 \times 10^{-12} \mathrm{~s}^{-2}$, and the contour $8.0 \times 10^{-12} \mathrm{~s}^{-2}$ is added for convenience; $(b) \mathrm{CI}=0.4 \times 10^{-12} \mathrm{~s}^{-2}$. The boundary points, all with zero variance, are not shown.

where $\sigma_{f}=\left\langle f^{2}\right\rangle$ is the $f$-variance. The time mean eddy forcing is characterized by the dipole pattern that enhances the main eastward jet in the upper ocean, but drives the opposite current in the deep ocean (figure 1). Below, properties of $\sigma_{f}, R_{T}, R_{H}$, and $R_{V}$, are analysed statistically and dynamically (i.e. by calculating the corresponding non-eddy-resolving solutions).

The $\sigma_{f}$ is spatially inhomogeneous (figure 3 ): its maximum values are located in the western part of the basin and in the upper ocean; in the east, dominated by the Rossby waves, the variance is small. Instantaneous spatial patterns of the eddy forcing are complex, and it is not easy to associate them with flow patterns (figure 2). The dominant length scale of the eddy-forcing pattern increases gradually from west to east. The third- and higher-order statistical moments of the eddy forcing indicate strongly non-Gaussian, that is, intermittent, statistical distributions. In particular, the prevailing large and positive values of the kurtosis, that is, the fourth-order moment non-dimensionalized by the variance, indicate enhanced probability of both very strong and weak events. The kurtosis values of more than 10 are found around the subtropical western boundary current and its eastward jet, and near the northern and southern boundaries. In the random-forcing model, the intermittency is neglected, for simplicity.

\subsection{Temporal correlations}

It is found that $R_{T}\left(\tau, x_{0}\right)$ decays and oscillates for all $\boldsymbol{x}_{0}$ (figure 4). The minimum value of $R_{T}$ averaged over the western third of the basin is about -0.2 , and the corresponding value for the eastern third of the basin, where $R_{T}$ has more pronounced oscillations, is about -0.65 . In the western basin, dominated by relatively localized eddies generated by the western boundary current and eastward jet, $R_{T}$ decays below the 0.1 threshold on a time scale of 1 month. In the eastern basin, this time scale is about 3 months, which is the time scale of the first baroclinic Rossby-wave mode. This increase of the decay time scale is associated with broader spatial correlations (§3.2).

The $R_{T}$ oscillates on the time scale of about 40 days, but in the western basin this oscillation is masked by rapid decay of correlations. Over this time scale, a 

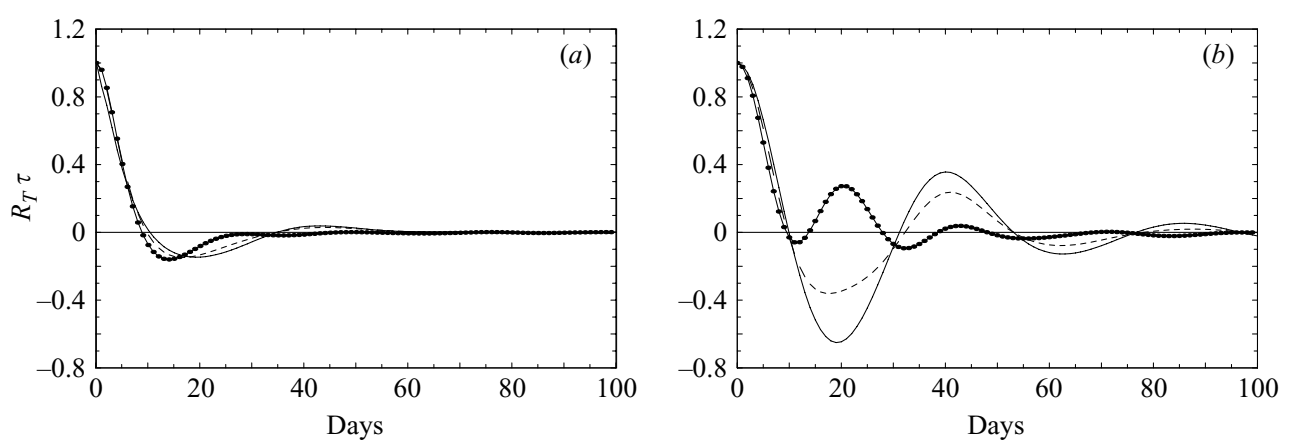

Figure 4. The area-averaged $R_{T}(\tau)$ in the $(a)$ western $1 / 3$ and $(b)$ eastern $1 / 3$ of the basin. The upper-, middle- and deep-ocean $R_{T}(\tau)$ are plotted by the solid, dashed and dotted lines, respectively.

fluctuation in the form of the first baroclinic Rossby-wave mode propagates over 3.5 deformation radii, which is a typical eddy scale. The $R_{T}$ based on the verticalmode projection of the eddy forcing shows that the barotropic component contains a signal with approximately half this time scale of about 20 days. The fast barotropic component rides on the slow baroclinic component, and therefore the deep-ocean $R_{T}$, which is dominated by the barotropic mode, is strongly asymmetric (i.e. with the enhanced second positive lobe), with 20-day oscillation superimposed on the 40-day one (figure $4 b$ ). The mechanism of the excitation of the barotropic mode excitation is unclear, but the presence of the signal is not surprising, given the variety of organized propagating patterns observed at similar Re (Berloff \& McWilliams 1999a). Overall, this behaviour illustrates that the eddy forcing can be affected by new modes excited by the non-eddy-resolving dynamics.

Spatial distribution of the integral correlation time,

$$
T_{I N T}\left(x_{0}\right)=\int_{0}^{\infty} R_{T}\left(\tau, x_{0}\right) \mathrm{d} \tau,
$$

is difficult to explain (figure 5). In the upper-ocean western boundary currents and the main eastward jet, $T_{I N T}$ is relatively large (3-10 days), owing to the relatively weak negative lobe of $R_{T}$, and toward the east it gradually decays to about 1 day. In the deep ocean (the middle layer is the transition zone), the situation is the reverse: because of the barotropic-mode contribution, $T_{I N T}$ increases from 4 days near the western boundary to more than 10 days near the eastern one.

\subsection{Horizontal correlations}

Spatial correlations of the eddy forcing are characterized by the single central maximum at $\boldsymbol{x}_{0}$ and by oscillations with alternating sign. The $R_{H}$ shapes can be classified into three types (figure 6): (a) approximately monotonic and isotropic mesoscale pattern found in the western basin, except for the upper-ocean eastward jet; $(b)$ oscillating and anisotropic mesoscale pattern in the upper-ocean eastward jet; and $(c)$ wavelike and anisotropic large-scale pattern in the eastern basin. The relatively long-living and approximately axisymmetric vortices generated in the main western boundary current and eastward jet yield type $(a)$. Type $(b)$ is due to the relatively short-living small mesoscale vortices (not shown) generated by the strongly sheared eastward jet with very large cross-jet potential-vorticity gradient (i.e. by the KelvinHelmholtz instability). These vortices, occasionally, during the events of smooth, 
(a)

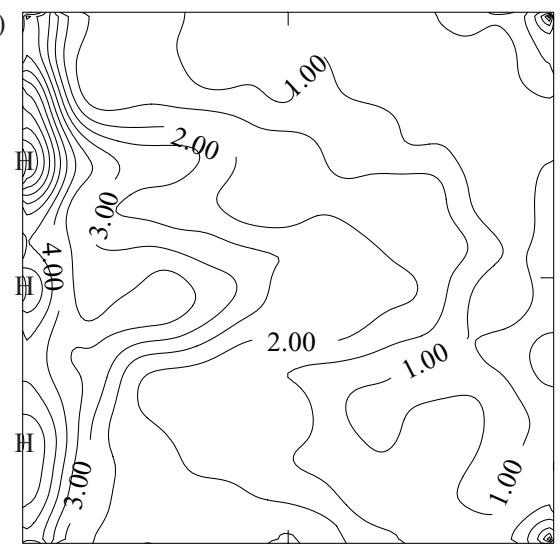

(c)

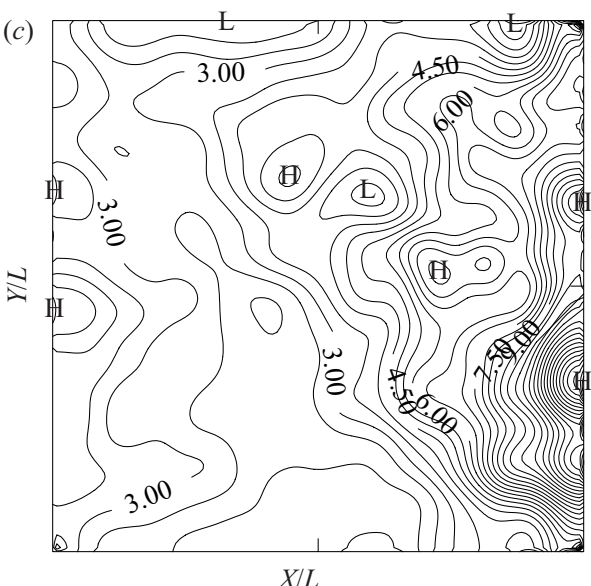

(b)

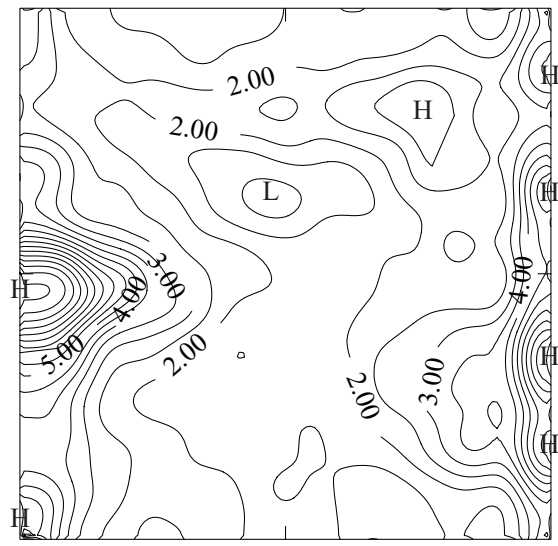

Figure 5. $T_{I N T}(x, y)$ in the $(a)$ upper, $(b)$ middle and $(c)$ deep ocean $(\mathrm{CI}=0.5$ days; some of the high and low extrema are indicated with the corresponding symbols).

that is, uninterrupted by large vortices, boundary-layer separation, appear in the form of relatively regular vortex chains. Thus, the alternating-sign pattern (figure $6 b$ ) is due to the advection of large-amplitude positive and negative potential-vorticity anomalies. Type $(c)$ is formed by the Rossby waves, therefore it is characterized by large oscillations, which are similar to those observed by Jayne et al. (1996) in a zonal-jet model. Type $(c)$ is also characterized by long-range positive correlations along the basin boundaries. The propagating nature of the signal that forms $(c)$ is evident in the delayed-time correlation function,

$$
\tilde{R}_{H}=\frac{\left\langle f\left(t, \boldsymbol{x}_{0}\right) f\left(t+\tau, x, y, z_{0}\right)\right\rangle}{\sigma_{f}^{1 / 2}\left(\boldsymbol{x}_{0}\right) \sigma_{f}^{1 / 2}\left(x, y, z_{0}\right)},
$$

which shows westward propagation of the coherent signal. The $\tilde{R}_{H}$ of type $(a)$ exhibits westward propagation of the barotropic component on the time scale of 1 month, but time-delayed correlations of type $(b)$ disappear within a few days.

The zonal, $L_{H x}$, and meridional, $L_{H y}$, correlation length scales are defined as such distances away from $\boldsymbol{x}_{0}$, over which $R_{H}$ decays to 0.3 ; the isotropic radius of correlation is defined as $L_{H}=\left(L_{H x}+L_{H y}\right) / 2$; and the correlation anisotropy coefficient is defined as $A_{H}=L_{H y} / L_{H x}-1$. The $L_{H}$ increases from the subtropical 

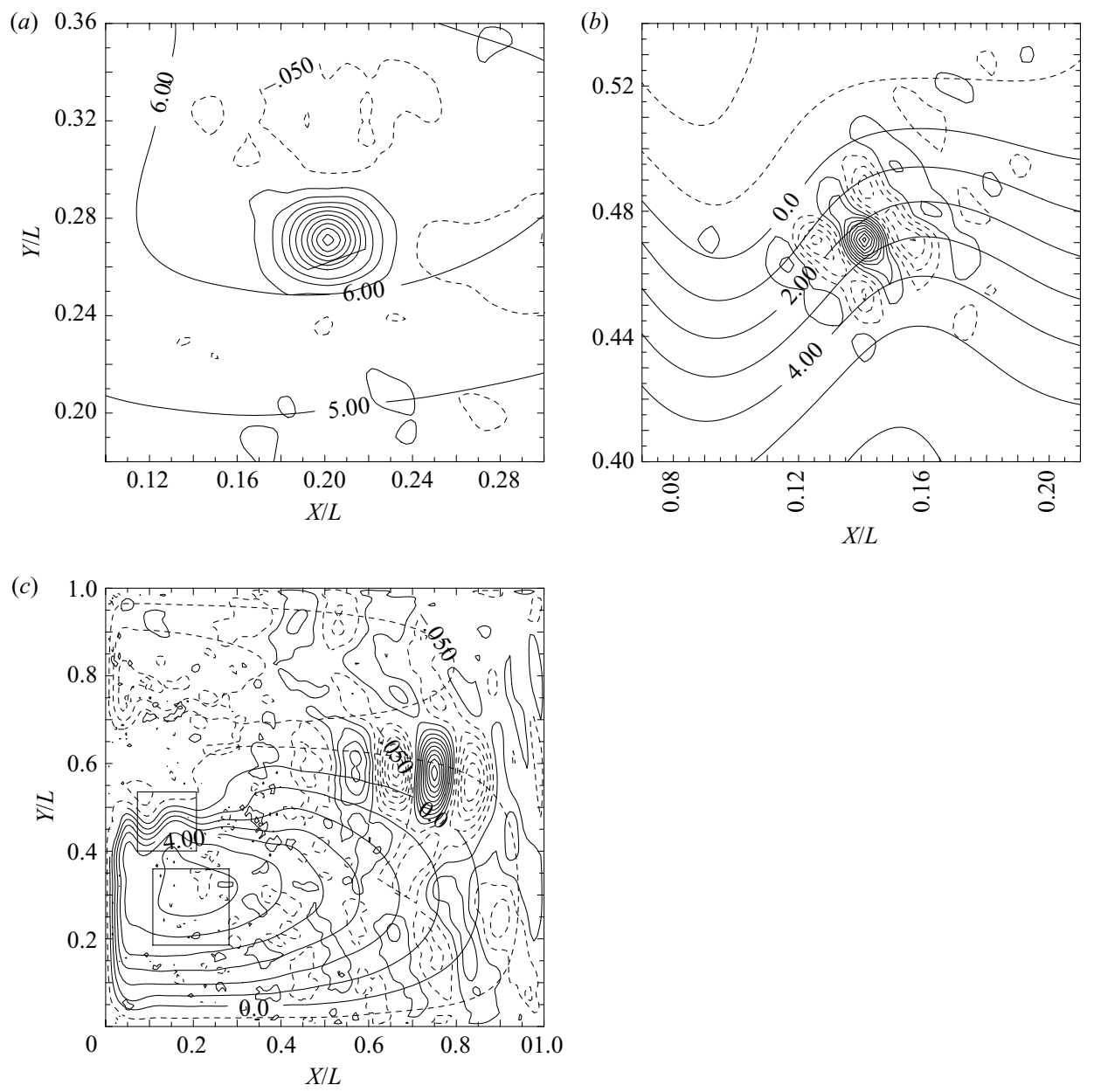

FIGURE 6. The upper-ocean $R_{H}$ for the reference location, $\left(x_{0} / L, y_{0} / L\right), \quad\left(0<x_{0}<L\right.$, $\left.0<y_{0}<L\right)$ at $(a)(0.20,0.28),(b)(0.15,0.48)$ and $(c)(0.76,0.56)(\mathrm{CI}=0.1$; and the contour levels run from -0.95 to 0.95$)$. The small squares in $(c)$ outline the regions zoomed out in (a) and $(b)$. The corresponding time-mean velocity streamfunction is shown with $\mathrm{CI}=$ $10^{4} \mathrm{~m}^{2} \mathrm{~s}^{-1}$.

western boundary current, where it is less than $40 \mathrm{~km}$, toward the east and north (figure $7 a$ ). In the eastern basin, it varies from 100 to $300 \mathrm{~km}$, with an average value of about $250 \mathrm{~km}$. The $A_{H}$ is small in the western basin, but it is large in the eastern basin (figure $7 b$ ), where $L_{H y}$ is about twice as long as $L_{H x}$, owing to the asymmetry introduced by the Rossby waves. The barotropic and baroclinic components of the eddy forcing have significant spatial correlations over the same length scales, except for the eastern basin, where the barotropic correlation (hence, the deep-ocean correlation) decays over the distance twice as short as that of the baroclinic correlation (the faster decay does not affect the wavelength). To summarize, both temporal and horizontal correlation patterns of the eddy forcing are set by the barotropic (in spite of its relatively small contribution to the streamfunction variance) and not baroclinic component, because the barotropic waves more efficient decorrelators. 


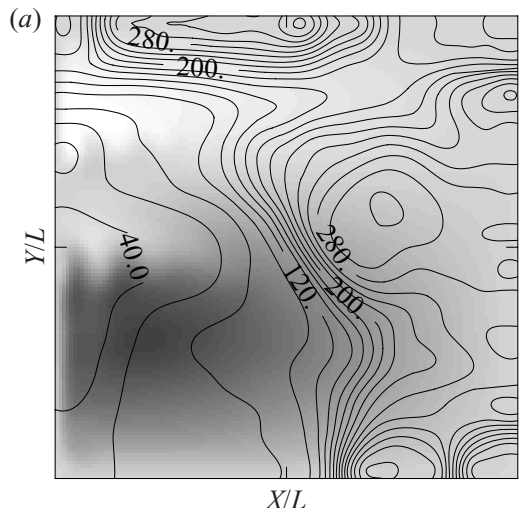

$-4$ (b)

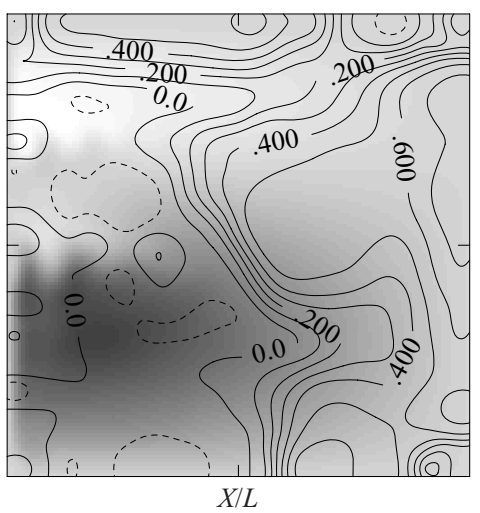

$+10$

FIgURE 7. The upper-ocean distributions of $(a) L_{H}(\mathrm{CI}=20 \mathrm{~km})$ and $(b) A_{H}(\mathrm{CI}=0.1)$. The corresponding time-mean velocity streamfunction is shown with the grey colour scale and $10^{4} \mathrm{~m}^{2} \mathrm{~s}^{-1}$ units.

(a)

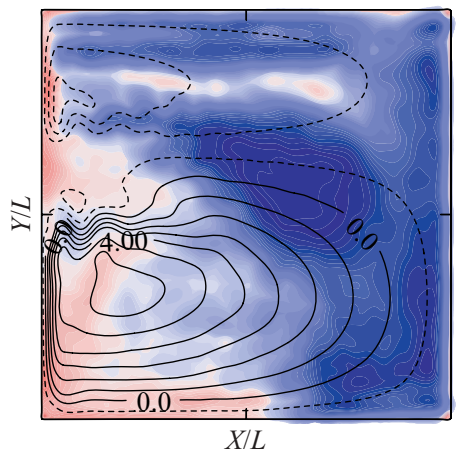

(b)

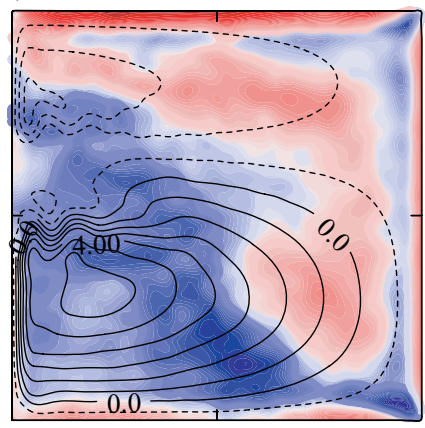

$X / L$ (c)

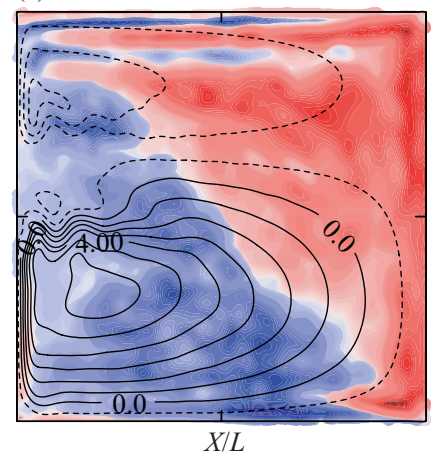

$-1$

$+1$

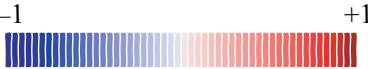

FIGURE 8. Correlations between the different isopycnal-layer components of the eddy forcing: (a) $C\left(f_{1}, f_{2}\right),(b) C\left(f_{2}, f_{3}\right)$ and $(c) C\left(f_{1}, f_{3}\right)$. The upper-ocean time-mean velocity streamfunction is shown for convenience with the contours $\left(C I=10^{4} \mathrm{~m}^{2} \mathrm{~s}^{-1}\right)$.

\subsection{Vertical correlations}

The three-layer vertical stratification does not properly resolve the actual oceanic $R_{V}$, therefore the focus here is on the most basic properties only. Overall, the eddy forcing is substantially correlated in the vertical direction (figure 8). Relative to fluctuations of $\psi$, the eddy forcing is characterized by very weak barotropic, by the weakened first baroclinic, and by the dominant second baroclinic components. This redistribution of correlations between the vertical modes occurs because, typically, the lower the order of the mode, the larger is its horizontal length scale; therefore, the lower modes are better captured by the reduced dynamics, and the corresponding residual eddy forcing is weaker. The second-mode dominance is equivalent to substantial negative correlations between the upper and middle layers, and to substantial positive 
correlations between the upper and deep layers (the vertical mode structure is not shown). Around the subtropical western boundary current and eastward jet, the eddy forcing is concentrated in the upper ocean - this behaviour is associated with positive correlations between all of the vertical modes. In the central and southern parts of the subtropical gyre, the first baroclinic mode dominates; hence, there is substantial positive correlation between the upper and middle layers, and there is negative correlation between the upper and deep layers.

\section{Modelling random forcing}

Here, the random-forcing model is formulated and tested. The flow rectification mechanism is described in $\S 4.1$; and the way the temporal evolution and spatial correlations are represented is given in $\S \S 4.2$ and 4.3. The roles of the spatial resolution, variance and spatial correlations are studied in the next three sections.

\subsection{Physical mechanism: rectified flow driven by fluctuating forcing}

Purely fluctuating eddy forcing is capable of driving rectified (i.e. with a non-zero timemean component) large-scale flows. Here, the principal physical mechanisms behind the rectification phenomenon are discussed. Two regimes of rectification are relevant to the double gyres, and both of them result in formation of zonal currents (Rhines $1975,1994)$. Let us consider potential-vorticity dynamics forced by the localized zero-mean external forcing, $\Phi(t, \boldsymbol{x})$, with (positive) amplitude $A$ :

$$
\frac{\mathrm{d} q}{\mathrm{~d} t}+\beta v=A \Phi(t, \boldsymbol{x}) .
$$

By integrating (13) over a short time interval and by multiplying the result by the flow velocity, we obtain:

$$
\delta q v=\left(A \int \Phi \mathrm{d} t-\beta \delta y\right) v .
$$

If $A \rightarrow 0$, then, taking into account that $v=\delta y / \delta t$, we average over the ensemble of realizations (here, the ensemble averaging is denoted by angle brackets) and obtain:

$$
\langle\delta q v\rangle=-\frac{\beta}{2} \frac{\mathrm{d}}{\mathrm{d} t}\left\langle y^{2}\right\rangle .
$$

If there is irreversible Lagrangian dispersion of the fluid elements, then there is the asymptotic diffusive limit, in which: $\left\langle y^{2}\right\rangle \sim t$. In this limit, the right-hand side of (15) is negative, hence the ensemble-average meridional flux of $q$, which is equivalent to the Eulerian flux of $q$, is negative. This flux results in the westward acceleration of the background flow. In short, this flow regime is a result of the meridional mixing and partial homogenization of the absolute potential vorticity. The second regime occurs when the forcing is sufficiently strong and $v$ is substantially correlated with it (that is, $\left\langle v \int \Phi \mathrm{d} t\right\rangle>0$ ), which is a typical phenomenon for the $\beta$-plane dynamics, (13), in the near-Sverdrup regime. Then, the meridional $q$-flux is positive, hence, it drives the eastward current. Both regimes can be present simultaneously when the forcing is strong around some latitude, but weak elsewhere. This is the case in the double-gyre solution which is characterized by the enhanced eddy-forcing variance around the main eastward jet (figure 3). As a result, the flow rectification consists of the cyclonic/anticyclonic recirculations, which are discussed further below, to the north/south of the eastward jet. 


\subsection{Modelling temporal evolution}

Although the observed $R_{T}(\tau)$ is complex, it can be approximated by the second-order autoregressive (AR-2) process, which is capable of simulating exponentially decaying oscillations (Box, Jenkins \& Reinsel 1994). The AR-2 process for function $f$ is defined at discrete times separated by the timestep, $\mathrm{d} t$ :

$$
f(t)=\phi_{1} f(t-\mathrm{d} t)+\phi_{2} f(t-2 \mathrm{~d} t)+\mathrm{d} W,
$$

where $\mathrm{d} W$ is a random increment (Wiener process). Given time scales of the exponential decay, $\theta$, and the oscillation, $T$, the AR-2 coefficients are:

$$
\phi_{1}=2 \exp (-\mathrm{d} t / \theta) \cos (2 \pi \mathrm{d} t / T), \quad \phi_{2}=-\exp (-2 \mathrm{~d} t / \theta) .
$$

The autocorrelation function for the AR-2 process is:

$$
R_{T}(\tau)=\exp (-\tau / \theta) \sin \left(\frac{2 \pi}{T} \tau+\Phi\right)[\sin \Phi]^{-1},
$$

where the phase shift, $\Phi$, is found from the equation:

$$
\tan \Phi=\frac{1+\exp (-2 / \theta)}{1-\exp (-2 / \theta)} \tan \left(\frac{2 \pi}{T}\right) .
$$

The first-order autoregressive process (AR-1), which is given by

$$
\phi_{1}=\exp (-\mathrm{d} t / \theta), \quad \phi_{2}=0, \quad R_{T}(\tau)=\exp (-\tau / \theta),
$$

is a more simple model for the time evolution - it captures only exponential decay. Here, either model is integrated with $\mathrm{d} t=0.2$ day.

By considering the non-eddy-resolving model with idealized random-forcing functions, it is found that $T_{I N T},(11)$, is one of the most important eddy-forcing parameters. For the AR-2 process, it is:

$$
T_{I N T}=\theta\left[1+\frac{2 \pi \theta}{T \tan \Phi}\right]\left[1+\left(\frac{2 \pi \theta}{T}\right)^{2}\right]^{-1},
$$

and for the AR-1 process, it is:

$$
T_{I N T}=\theta
$$

In the first experiment, the observed $R_{T}$ is approximated with: $T_{I N T}=3$ and $T=$ 40 days. These values are taken on the basis of the analysis presented in $\S 3$. The functional dependence of $T_{I N T}$ on $\theta$ and $T$ is such that the same value of $T_{I N T}$ can be generally achieved for two cases: without and with the change of sign in $R_{T}$ (figure 9). The values of $\theta$, found numerically as roots of (21), are $\theta_{1}=1.6993297$ and $\theta_{2}=25.2987717$ days, and the corresponding $R_{T}$ are very different from each other (figure 9). In the second experiment, the AR-1 process is used with three values of $T_{I N T}=\theta: 3.3,10$ and 30 days. It is found that, in the realistic range of the eddy variance and space correlations, the time-mean non-eddy-resolving solutions are largely insensitive to the oscillating structure of $R_{T}$, because the difference between the $\theta_{1}$ and $\theta_{2}$ solutions is negligible. On the other hand, the flow amplitude, both the time-mean and instantaneous, monotonically increases with the above $T_{I N T}$ values, each time by a factor of 2.7 .

\subsection{Modelling spatial structure}

The method of constructing space-correlated random functions is based on calculating an $N$-dimensional random vector (for $N$ spatial grid points) and on multiplying it 

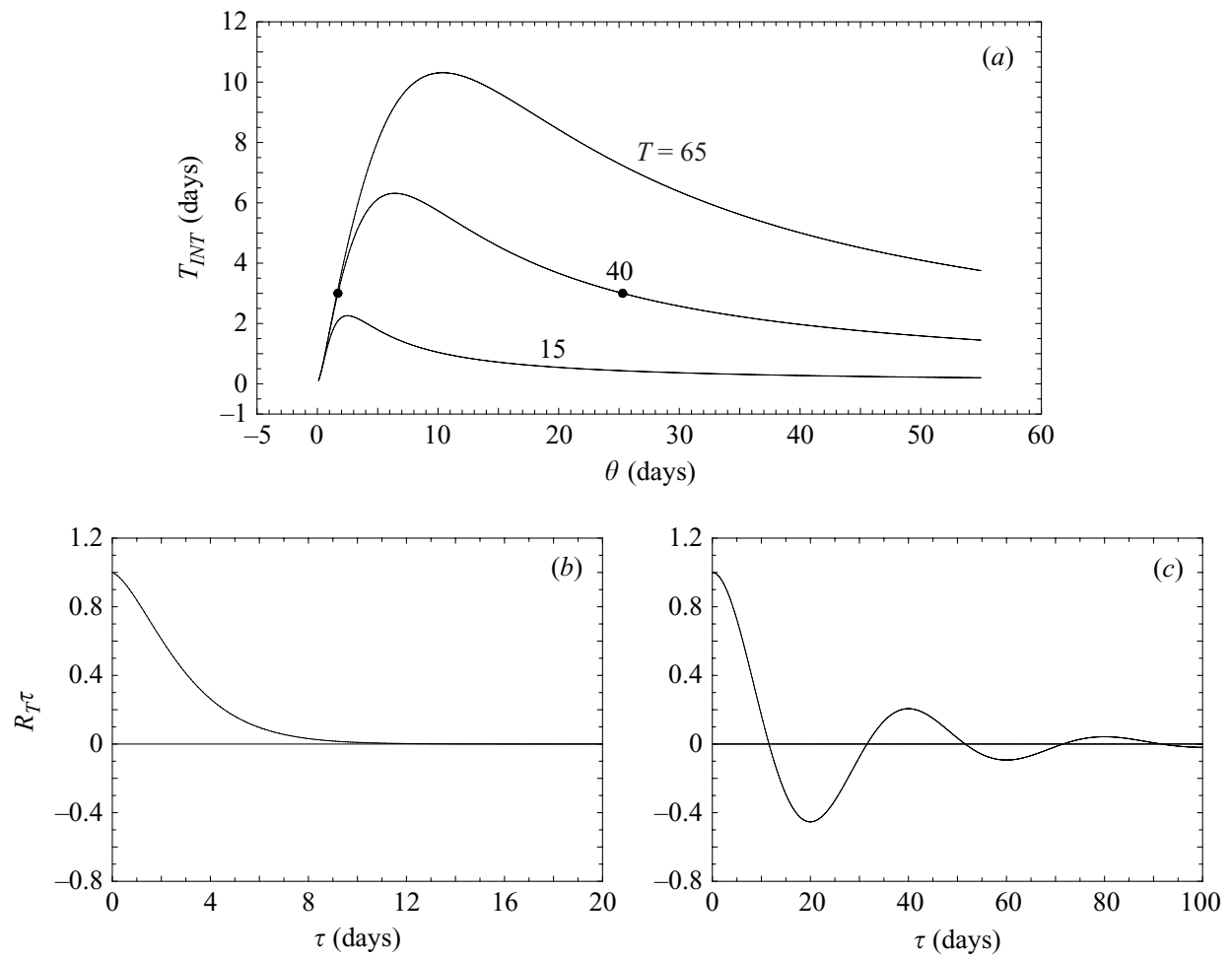

FiguRE 9. (a) $T_{I N T}(\theta)$ for $T=15,40$ and 65 days. The pair of $R_{T}(\tau)$ for $T$ and $T_{I N T}$ equal to 40 and 3 days, respectively, for the parameters: $(b) \theta=1.7$ and $(c) \theta=25.3$ days. In $(a)$, the parameters $\theta$ corresponding to the $R_{T}(\tau)$ shown in $(b)$ and $(c)$ are indicated by the filled circles.

with the corresponding transformation matrix (Appendix B). For the stationary statistics, the transformation matrix is calculated just once. Overall, the method is intentionally local rather than spectral, because inhomogeneity of the spatial correlations is strong. The method is further optimized by using a spatially nonuniform grid, with low resolution in parts of the basin characterized by weak gradients of the space correlation function (Appendix B). Three grids are used, with uniform low, non-uniform standard, and non-uniform high resolutions. The vertical correlations are incorporated into the random-forcing model by the same method, by finding the vertical transformation matrix, separately.

\subsection{Random-forcing solutions: resolution}

The analysed random-forcing solutions differ in terms of the spatial resolution, spatial correlations and variance. Here, the temporal evolution of the forcing is modelled as the AR-1 process, with distribution of the exponential decay time scale given by the integral time scale (figure 5). All of the solutions are routinely found for the model configurations with and without the wind and time-mean eddy forcing, but only the most informative results are reported here. Below, the focus is on situations with purely fluctuating forcing. First, the benchmark STANDARD solution and the analogous solutions with the lower and higher resolutions are looked at. By using the moderately coarsened grid as the standard one, the fact is emphasized that computational expenses required for reasonably good-quality solutions can be 

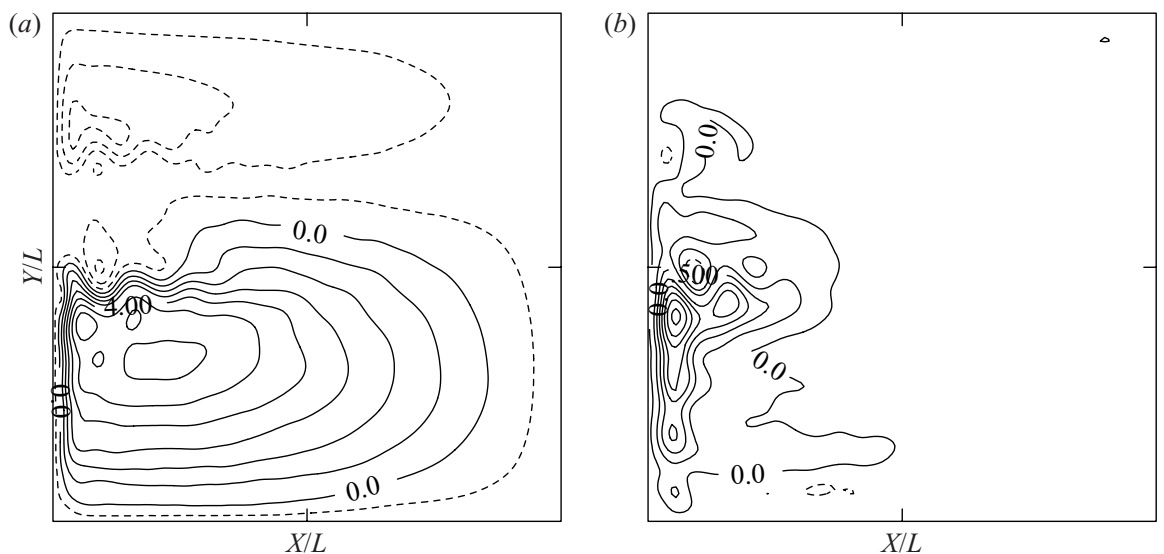

FIGURE 10. (a) Upper- and (b) deep-ocean velocity streamfunctions of the STANDARD solution of the non-eddy-resolving model. (a) $\mathrm{CI}=10^{4} \mathrm{~m}^{2} \mathrm{~s}^{-1},(b) 0.25 \times 10^{4} \mathrm{~m}^{2} \mathrm{~s}^{-1}$.

moderate. In terms of the random-forcing properties, the corresponding solutions are labelled as:

1. STANDARD: the spatially inhomogeneous variance; non-uniform standard grid; empirical spatial correlations;

2. LR-STANDARD: the same, but with the low-resolution grid;

3. HR-STANDARD: with the high-resolution grid.

In the western basin, the STANDARD forcing replaces $3 / 4$ of the original degrees of freedom with spatially interpolated values (Appendix B) - this, effectively, increases correlation length scales in the subtropical western boundary current and its eastward jet and, therefore, enhances the dynamic flow response. In other words, the forcing generated on a coarsened grid does not properly resolve the small-scale features of the original-grid covariance. Some of the small-scale fluctuations are anti-correlated, therefore they largely compensate each other in terms of the dynamic response. This compensation is unambiguously demonstrated by adding small regions of negative forcing amplitude to the west and east of the localized, spatially coherent forcing placed in the centre of the basin. The amplifying effect of the covariance coarsening can be compensated for by reducing $\sigma_{f}$. Here, the reduced $\sigma_{f}$ is calculated by applying the grid-scale 9-point Gaussian filter to the instantaneous fields of the diagnosed eddyforcing history $\uparrow$ : this results in a similar strength of the flow rectification, as in the reference solution.

Overall, climatology of the STANDARD solution (figure 10) is qualitatively similar to that of the reference solution (figure 1) - this important result proves that actual eddy forcing can be simulated as a random process. The main deficiency of the STANDARD and other solutions is that they slightly underestimate flow in the subtropical western boundary current. As a result, the southern inertial recirculation does not extend to the southern boundary but, instead, concentrates near the eastward jet (figure 11a). It is plausible that the underestimate in the subtropical

$\dagger$ There is a general warning against calculating covariance of the filtered field, because it is likely not to be a positive-definite one, as required by the method of solving the $S$-problem. In general, the best way to reduce the number of the degrees of freedom contained in the random forcing is to further reduce the whole dynamic model. 

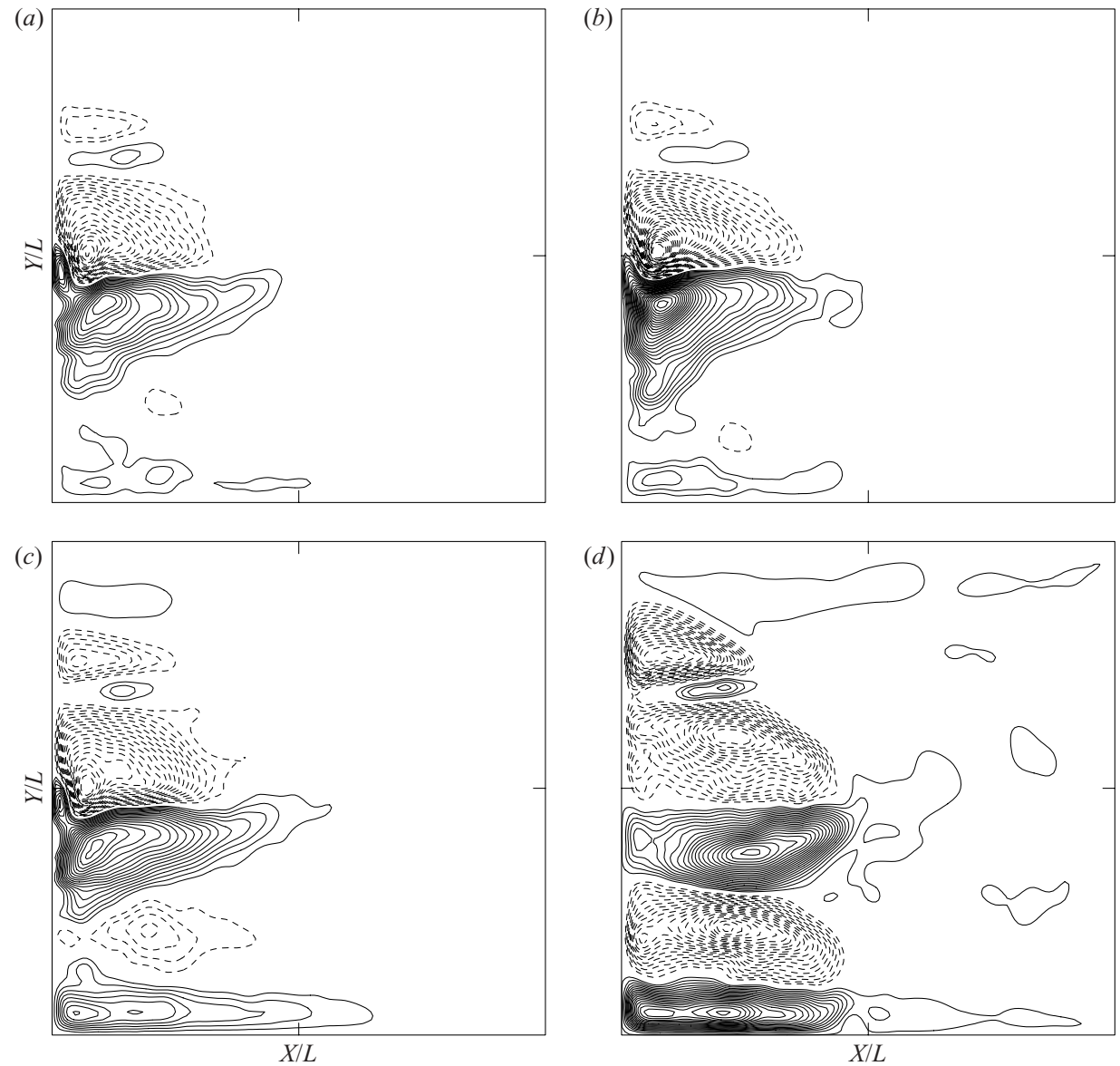

FIGURE 11. Upper-ocean streamfunctions of the non-eddy-resolving model solutions driven without wind, by purely fluctuating random forcing: (a) STANDARD, (b) MIXED, (c) VERT-1 and $(d)$ VAR-WEST-HOMOG $\left(\mathrm{CI}=10^{3} \mathrm{~m}^{2} \mathrm{~s}^{-1}\right)$.

western boundary current is a result of the neglected joint space-time correlations, which are associated with the strong downstream-propagating near-boundary vortices (Berloff \& McWilliams 1999b). The neglected intermittency of the eddy forcing can be another reason. Another drawback of the STANDARD solution is that the northern recirculation is too compact, but this problem is fixed in the HR-STANDARD solution - this is the only substantial improvement of the higher resolution. The LR-STANDARD solution is obtained with $\sigma_{f}$ reduced by smoothing the noise until it yields the correct transport in the eastward jet. (The result is largely insensitive to the smoothing algorithm, and here it is achieved by applying the grid-scale 25point Gaussian filter twice.) The LR-STANDARD solution is a smoother version of the STANDARD solution, but it has an eastward jet only half as long and an approximately symmetric and relatively localized recirculation pattern. On the one hand, the LR-STANDARD solution illustrates the ultimate robustness of the eastward-jet enhancement; on the other hand, it is a poor-quality solution that indicates the practical limit of the grid coarsening. 


\subsection{Random-forcing solutions: spatial correlations}

Random forcing of each of the following solutions (denoted by the corresponding symbol) has only one spatial-correlation property that is different from the STANDARD case.

4. GAUSS-STANDARD: gaussian approximation of the spatial correlations.

5. GAUSS-SHORT: very compact Gaussian spatial correlations (10 km length scale).

6. MIXED: linear combination of the spatial correlations from the STANDARD and GAUSS-STANDARD solutions with the variance weights of $4 / 5$ and $1 / 5$, respectively.

7. VERT-0: no vertical correlations between the isopycnal-layer random forcing functions.

8. VERT-1: absolute positive correlation between the isopycnal-layer forcing functions.

The complex spatial correlations of the observed eddy forcing are approximated with a much simpler Gaussian shape of the correlation function (9):

$$
R_{H}\left(\boldsymbol{x} ; \boldsymbol{x}_{0}\right)=\exp \left[\frac{\left(x-x_{0}\right)^{2}}{X_{m}^{2}}+\frac{\left(y-y_{0}\right)^{2}}{Y_{m}^{2}}\right],
$$

where $X_{m}$ and $Y_{m}$ are the Gaussian correlation length scales defined at the middle point, $\left(\boldsymbol{x}-\boldsymbol{x}_{0}\right) / 2$. The rotational symmetry, $R_{H}\left(\boldsymbol{x} ; \boldsymbol{x}_{0}\right) \equiv R_{H}\left(\boldsymbol{x}_{0} ; \boldsymbol{x}\right)$, is automatically satisfied, because of the mid-point definition. The covariance matrix corresponding to (23) is positive-definite $\dagger$, as required by the method of solving the spatial-correlation problem (Appendix B). The correlation length scales estimated from the diagnosed eddy forcing (figure 6) are used for $X_{m}$ and $Y_{m}$. The GAUSS-STANDARD solution overestimates the rectified flow recirculations by a factor of 2.5 - this suggests that the structure of the spatial correlations and, in particular, the ubiquitous negative correlations are very important. The overestimate by a factor of more than 2 is found in the GAUSS-SHORT solution, in which the forcing is virtually uncorrelated on the grid interval. Hence, the correlation length scale reduction cannot compensate for the negative correlations. If the forcing is defined as the linear combination of the Gaussian and STANDARD forcings (i.e. the MIXED case), then $20 \%$ contribution of the Gaussian component results in $40 \%$ enhancement of the flow amplitude (figure 11b). Thus, the MIXED solution shows that even small corruption of the negative correlations results in qualitative error.

The vertically uncorrelated forcing (VERT-0) yields only small differences in the flow, if compared to the STANDARD case. This is because in the most energetic subtropical western boundary current/eastward jet region, the vertical eddy-forcing correlations are small (figure 8). On the other hand, the absolutely correlated forcing (VERT-1) yields noticeable enhancement of the rectified recirculations around both eastward jets and of the anticyclonic cell along the southern boundary (figure 11c). This enhancement is associated with substantial barotropization of the flow, and the question of why the barotropic mode rectifies more efficiently requires further study.

\subsection{Random-forcing solutions: variance}

Here, the dynamical role of the spatial inhomogeneity of $\sigma_{f}$ is analysed. In the following solutions, the forcing variance is non-zero only in certain region.

$\dagger$ Here, general sign-changing $R_{H}$ are not studied, because an arbitrary shape of $R_{H}$ does not guarantee positive definiteness. 
9. VAR-UPPER: in the upper ocean.

10. VAR-DEEP: in the deep ocean.

11. VAR-WBC: in the western boundary current.

12. VAR-WEST: in the western basin.

13. VAR-EAST: in the eastern basin.

14. VAR-WEST-HOMOG: as VAR-WEST, but with the locally averaged homogeneous value.

The VAR-UPPER and VAR-DEEP solutions show that the flow is dominated by the upper-ocean forcing. In the VAR-UPPER case, it is found that the upper- and deep-ocean responses are about $10 \%$ and $50 \%$ weaker, respectively, and the deep ocean develops the time-mean recirculations, which are more similar to the upperocean recirculations than to those of the STANDARD solution. In the VAR-DEEP case, it is found that the upper-ocean response is just $13 \%$ of the STANDARD one, and flow in the main recirculations is opposite to the STANDARD one. The other solutions are obtained by partitioning the basin into three regions characterized by the upper-ocean $\sigma_{f}$ (figure 3): $\sigma_{f}>10 \times 10^{-12} \mathrm{~s}^{-2}$ (VAR-WBC), $\sigma_{f}>2.5 \times 10^{-12} \mathrm{~s}^{-2}$ (VAR-WEST), and $\sigma_{f}<2.5 \times 10^{-12} \mathrm{~s}^{-2}$ (VAR-EAST). Overall, the corresponding solutions show that the flow is dominated by the western-basin eddy forcing, and the main eastward-jet recirculations are dominated by the forcing which is even more limited to the western boundary-current region. The VAR-WBC forcing yields the main recirculations with $90 \%$ of the STANDARD strength, and the VAR-WEST forcing yields qualitatively correct flow in the western part of the basin. Thus, the strength of the eastward jet is largely controlled by the up-stream eddy forcing.

The VAR-EAST solution is characterized by the set of the time-mean zonal jets with the meridional scale of about $280 \mathrm{~km}$. The alternating jets correspond to the rectified flow regime driven by homogeneous variance (Rhines 1994). Finally, the importance of the spatial inhomogeneity is illustrated by averaging the upper-ocean $\sigma_{f}$ over the WEST region (the VAR-WEST-HOMOG solution) - this is an attempt to reduce $\sigma_{f}$ to some universal value. Although the resulting time-mean flow still possesses some qualitatively correct features, its main recirculations (and the eastward jet) are too weak, and all the other recirculations are too strong (figure $11 d$ ); hence, the idea of the universal value fails. Finally, the VAR-WEST-HOMOG solution suggests that the absence of the eastward-jet enhancement in Seidov \& Marushkevich (1992) is a result of the homogeneity of the random forcing.

\section{Summary and discussion}

The approach taken in this paper is a combination of mathematical modelling and dynamical analysis of the eddy effects on the large-scale ocean circulation. The main result of this work is to demonstrate that the large-scale flow can be simulated by a non-eddy-resolving dynamical model driven by a random process that represents actual eddy forcing. This approach is an alternative to the common turbulent diffusion approach, and in the ocean gyres it allows us to simulate important anti-diffusive eddy effects.

\subsection{Random-forcing modelling}

First, the eddy-forcing history is diagnosed from the eddy-resolving reference solution. This is done by the dynamical decomposition method, which ensures that the noneddy-resolving ocean model driven by the corresponding eddy-forcing history correctly approximates the reference solution. Here, the eddy-resolving grid contains $512 \times 512$ 
nodes with $7.5 \mathrm{~km}$ resolution, and the non-eddy-resolving grid contains $129 \times 129$ nodes with $30 \mathrm{~km}$ resolution. The diagnosed history and the corresponding non-eddyresolving solution are the starting points for further analysis and modelling.

First, it is found that nonlinearity of the non-eddy-resolving model is fundamentally important, because it is responsible for the flow rectification. Secondly, the eddyforcing statistics, which are treated as spatially inhomogeneous but stationary, are obtained, and, on the basis of them, the eddy forcing is modelled as a simple random process with non-zero time average. The diagnosed eddy forcing is substantially correlated over time intervals ranging from about 1 month in the western to more than 3 months in the eastern basin. In the west, the time scale is set by intense coherent vortices, and in the east, by the Rossby waves. In most of the basin, the corresponding autocorrelation function oscillates on the time scale of about 40 days, which is set by the barotropic Rossby waves. In the western basin, the horizontal spatial correlations of the eddy forcing are nearly isotropic and relatively localized, and in the eastern basin they are in the form of large-scale and anisotropic structures imposed by the Rossby waves. Typically, spatial correlations oscillate and change sign. The vertical correlations are dominated by high-order vertical modes in the east, and by surface-intensified fluctuations which contain a mixture of the modes, in the west. If the non-eddy-resolving grid is reduced to $65 \times 65$ nodes, then the pattern of the spatial and temporal correlations remains qualitatively the same, except for the short-scale horizontal correlations in the eastward-jet region, that become essentially unresolved.

It is shown, that the diagnosed time evolution of the random forcing can be approximately modelled by an autoregressive process, and spatial correlations can be introduced by the transformation matrix calculated from the eddy-forcing covariance.

\subsection{Dynamic response to the random forcing}

The benchmark solution correctly simulates the reference climatology; hence, the random-forcing approach works. This is so, because the large-scale flow rectifies and enhances the subtropical eastward jet. Next, non-eddy-resolving randomly forced solutions are analysed for different parameters of the forcing. It is found that the integral time scale is an important parameter, but the period of the oscillations of the autocorrelation function plays no significant role. The structure of horizontal spatial correlations is important: positive and negative correlations partially compensate each other in terms of the large-scale response. The vertical correlations are found to be relatively unimportant, but this can be a result of the poor vertical resolution. Spatial inhomogeneity of the eddy-forcing variance, which has maximum values in the subtropical western boundary current and its eastward-jet extension, is found to be very important because it qualitatively sets the large-scale flow pattern. Finally, in the numerical sensitivity analysis, coarsening the spatial resolution results in the more symmetric and more confined to the west rectified flow recirculations.

\subsection{Further developments}

The following developments of the results of this paper are anticipated. The random-forcing approach can be extended toward other geophysical flows. Other properties of the eddy forcing have to be statistically and dynamically analysed: full, three-dimensional spatial correlations, without separation into the horizontal and vertical components; combined space-time correlations, with emphasis on propagating components; details of the sign-changing spatial correlations; and intermittency. Also, the random-forcing model does not account for material transport induced by the eddies, therefore, it has to be complemented by a transport model which is likely to be 
non-diffusive on the intermediate time scales (e.g. Griffa 1996; Berloff \& McWilliams 2003). As the starting-point along this line, we have to analyse transports induced by the dynamically consistent eddy and large-scale components of the ocean gyres. Hopefully, the approach can be advanced even further, to primitive-equation general circulation models, but potential challenges along these lines are not yet known. In this case, we have to work with momentum rather than potential-vorticity eddy forcing, and the complementing transport models have to account for both temperature and salinity transports. So far, the main weakness of the random-forcing as well as the turbulent diffusion approaches, is uncertainty about relationships between parameters of the model for the eddy effects and large-scale flow patterns. Some progress can be made by studying long-range transport of the potential-vorticity fluctuations in the region that includes the subtropical western boundary current and its eastward-jet extension and by analysing low-frequency variability of the flow and its eddy forcing. Finally, the dynamical decomposition method can be optimized by incorporating a spatially non-uniform coarse grid, with finer resolution around the subtropical western boundary and its eastward-jet extension.

Fruitful conversations with Bill Dewar, Sergey Kravtsov, Michael McIntyre, Jim McWilliams, Joe Pedlosky and Bill Young are gratefully acknowledged. Suggestions made by anonymous reviewers also led to significant improvement of this work. Funding for this research was provided by NSF grants OCE 0091836 and OCE 0344094, by the Royal Society Fellowship, and by WHOI grants 27100056 and 52990035. The manuscript is WHOI contribution 10931.

\section{Appendix A. Parameters and solution of the eddy-resolving model}

Parameters of the eddy-resolving model are the following. The flow is driven at the surface by the asymmetric double-gyre zonal wind stress:

$$
\tau^{x}(y)=\tau_{0}\left[\cos \left(\frac{2 \pi(y-L / 2)}{L}\right)+2 \sin \left(\frac{\pi(y-L / 2)}{L}\right)\right],
$$

where $\tau_{0}=0.04 \mathrm{~N} \mathrm{~m}^{-2}$ and $L=3840 \mathrm{~km}$ is the size of the square basin (with $0 \leqslant y \leqslant L$, $0 \leqslant x \leqslant L)$. The lateral viscosity, that is, the eddy diffusivity, is $v=100 \mathrm{~m}^{2} \mathrm{~s}^{-1}$. The ocean is discretized vertically in three isopycnal layers with depths $H_{1}=200 \mathrm{~m}$, $H_{2}=1200 \mathrm{~m}$ and $H_{3}=2600 \mathrm{~m}$. The ratio of the density jumps across the layer interfaces is $\gamma=\left(\rho_{2}-\rho_{1}\right) /\left(\rho_{3}-\rho_{2}\right)=2$, which yields the first, $R d_{1}$, and second, $R d_{2}$, Rossby deformation radii of 52 and $30 \mathrm{~km}$. The stratification parameters in (2) are:

$$
\left.\begin{array}{l}
S_{i, 1}=f_{0}^{2}\left(H_{i} g \frac{\left(\rho_{i}-\rho_{i-1}\right)}{\rho_{1}}\right)^{-1} \quad(1<i \leqslant N), \\
S_{i, 2}=f_{0}^{2}\left(H_{i} g \frac{\left(\rho_{i+1}-\rho_{i}\right)}{\rho_{1}}\right)^{-1} \quad(1 \leqslant i<N),
\end{array}\right\}
$$

where $f_{0}=0.83 \times 10^{-4} \mathrm{~s}^{-1}$ is the mid-basin Coriolis parameter.

The eddy-resolving model operates at large Reynolds number,

$$
R e=\frac{U L}{v}=\frac{\tau_{0}}{\rho_{1} H_{1} \beta v} \approx 1000,
$$

where $U=\tau_{0}\left(\rho_{1} H_{1} L \beta\right)^{-1}$ is the upper-ocean Sverdrup velocity scale $\left(\approx 2.5 \mathrm{~cm} \mathrm{~s}^{-1}\right)$, and $\beta=2 \times 10^{-11} \mathrm{~m}^{-1} \mathrm{~s}^{-1}$. The horizontal grid resolution is uniform, with $513 \times 513$ grid 
points and $7.5 \mathrm{~km}$ intervals between them, so that the Munk length scale, $\delta_{M}=(v / \beta)^{1 / 3}$, is resolved by more than 2 grid points. The quasi-geostrophic equations (1) and (2) are discretized with the second-order finite differences and time stepped with the leapfrog scheme.

The upper-ocean time-mean circulation (figure 1) consists of the southern (subtropical) and northern (subpolar) gyres that fill about $2 / 3$ and $1 / 3$ of the basin, respectively, which is consistent with the wind stress pattern. The time-mean flow is characterized by the Sverdrup balance in the eastern part of the basin, and by the pair of western boundary currents and their eastward-jet extensions in the western part of the basin. The boundary currents do not merge with each other. This is a robust regime that appears at large $R e$ in the stratified and baroclinically unstable double-gyre flow with the no-slip boundary condition (e.g. Haidvogel, McWilliams \& Gent 1992; Berloff \& McWilliams 1999b; Siegel et al. 2001). In terms of the fluctuations, the basin can be partitioned into the more energetic 'western' part, characterized by strong vortices, and the less energetic 'eastern' part, dominated by the planetary waves (see Berloff \& McWilliams 2002 for details). In the deep ocean, the eddies are generally weaker, but they drive time-mean flow in the western basin.

\section{Appendix B. Cholesky factorization and generation of a non-uniform grid}

Here, the mathematical and numerical treatment of the spatial correlation problem is described. Given its covariance matrix, a space-correlated random-forcing vector, $f$, with $N$ degrees of freedom (for example, representing a $\sqrt{N} \times \sqrt{N}$ spatial grid), can be obtained from the space-uncorrelated, unit-variance noise, $g$, such that:

$$
\boldsymbol{g} \boldsymbol{g}^{T}=\boldsymbol{I}
$$

(here and below: the superscript, $T$, denotes transposition of the matrix; capitalletter symbols denote matrices; and $\boldsymbol{I}$ is the identity matrix). Each component of $g$ corresponds to an individual random time series generated at the corresponding grid point by an autoregressive process $\uparrow(\S 4.2)$. Let us assume that there is a linear relationship,

$$
\boldsymbol{f}(t)=\boldsymbol{L} \boldsymbol{g}(t)
$$

and the covariance matrix for $f$ is:

$$
\mathrm{C}=\left\langle\boldsymbol{f} \boldsymbol{f}^{T}\right\rangle
$$

Then, it is found that the transformation matrix, $\boldsymbol{L}$, is equal to the 'square root' of the covariance matrix, which is symmetric and positive definite:

$$
\boldsymbol{C}=\left\langle\boldsymbol{L} \boldsymbol{g}(\boldsymbol{L} \boldsymbol{g})^{T}\right\rangle=\left\langle\boldsymbol{L} \boldsymbol{g} \boldsymbol{g}^{T} \boldsymbol{L}^{T}\right\rangle=\boldsymbol{L}\left\langle\boldsymbol{g} \boldsymbol{g}^{T}\right\rangle \mathbf{L}^{T}=\mathbf{L} \mathbf{L}^{T}=\boldsymbol{L L}^{T} .
$$

$\boldsymbol{L}$ is found by factorizing positive definite $\boldsymbol{C}$ with the Cholesky algorithm (Press et al. 1992) and the 16-digit accuracy. Here, the correlation rather than covariance matrix is used for solving the $S$-problem - this allows us to look for solutions in terms of the unit-variance correlated noise, which is later multiplied by the spatially inhomogeneous square-root variance.

$\dagger$ If parameters of the process are spatially inhomogeneous, then solution of the $S$-problem shifts original parameters of individual time series. In the gyres, inhomogeneity of the integral time scale, $T_{I N T}$, is weak, therefore, shift of $T_{I N T}$ is negligible. In principle, this problem can be fixed by iterating the $S$-problem and correcting $T_{I N T}$. 


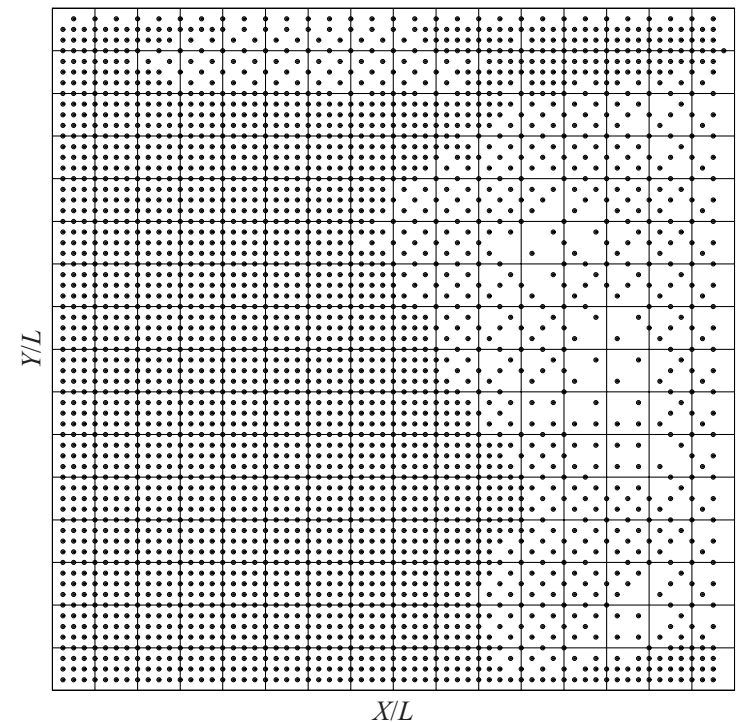

FIGURE 12. The non-uniform array of grid points used for the upper-ocean $S$-problem. Some of the original $129 \times 129$ grid lines are shown for convenience.

The factorization works, unless time series of the eddy-forcing history in the neighbouring grid points are too positively correlated, that is, if the points are too close to each other. In the ill-posed situation, the covariance matrix eigenvalues are close to zero (hence, the positive definiteness is in danger) and the method fails because of the numerical round-off error. To avoid the problem, the spatial-grid distances have to be made larger in places where correlations decay more slowly (e.g. in the eastern basin), and here this is achieved by the systematic rarefication of the original, $129 \times 129$ grid, without the boundary points. Also, the rarefication significantly reduces computational cost of the factorization. Here, three grids are implemented with uniform low, nonuniform standard, and non-uniform high resolutions.

For generating non-uniform grids, the original $129 \times 129$ grid points are grouped in several subsets, that is, in coarsened uniform grids, which are referred to as the $m$ thorder levels, L- $m$. Here, L- 1 is the $17 \times 17$ uniform grid, L-2 is the set of grid points added in the middle of the grid squares formed by the L-1 points, and L-3 is the set of grid points added in the middle of the grid squares (rotated by $45^{\circ}$ ) formed by the points from the combined, two lower levels, and the boundary points of L-3 are in the middle of the L-1 boundary points. Combined, the first three levels are equivalent to the $33 \times 33$ grid. When the next two levels, generated in the same way, are added, the grid is $65 \times 65$ points, L- 6 and L- 7 complete the original, $129 \times 129$ grid.

The uniform low-resolution grid consists of two levels. The other two grids are generated by the rejection algorithm: starting from the second level, a grid point is rejected if correlation of the $f$ between it and at least one of the nearest-neighbour points (from the same and lower levels) is greater than 0.82 and 0.74 in the upper and and deep ocean, respectively. Here, the correlation thresholds are found empirically. After $f$ is found at all not rejected points, its values at rejected points are restored by linear interpolation to the lower level, and from the $65 \times 65$ grid, $f$ is bicubically interpolated to the $129 \times 129$ grid. The non-uniform standard grid is generated with five levels, and in the upper ocean it contains $N=3027$ points (figure 12). The 
non-uniform high-resolution grid $(N=4239)$ is generated with seven levels in the western half of the basin, but in the eastern half it is equivalent to the standard grid. Deep-ocean non-uniform grids have $N$ which is $5 \%-10 \%$ smaller than in the upper ocean.

\section{REFERENCES}

Armenio, V., Piomelli, U. \& Fiorotto, V. 1999 Effect of the subgrid scales on particle motion. Phys. Fluids A 11, 3030-3042.

Berloff, P. 2004a Rectification of randomly forced flows. J. Mar. Res. (in press).

Berloff, P. $2004 b$ On dynamically consistent eddy fluxes. Dyn. Atmos. Oceans (in press).

Berloff, P. \& MCWilliams, J. 1999a Large-scale, low-frequency variability in wind-driven ocean gyres. J. Phys. Oceanogr. 29, 1925-1949.

Berloff, P. \& McWilliams, J. $1999 b$ Quasigeostrophic dynamics of the western boundary current. J. Phys. Oceanogr. 29, 2607-2634.

Berloff, P. \& McWilliams, J. 2002 Material transport in oceanic gyres. Part I: Phenomenology. J. Phys. Oceanogr. 32, 764-796.

Berloff, P. \& McWilliams, J. 2003 Material transport in oceanic gyres. Part III: Randomized stochastic models. J. Phys. Oceanogr. 33, 1416-1445.

Bleck, R., Dean, S., O’Keefe, M. \& Sawdey, A. 1995 A comparison of data-parallel and messagepassing versions of the Miami Isopycnic Coordinate Ocean Model (MICOM). Parallel Computing, pp. $1695-1720$.

Box, G., Jenkins, G. \& Reinsel, G. 1994 Time Series Analysis: Forecasting and Control. Prentice Hall, 598 pp.

Cessi, P. 1988 A stratified model of the inertial recirculation. J. Phys. Oceanogr. 18, 662-682.

Cessi, P., Ierley, G. \& Young, W. 1987 A model of the inertial recirculation driven by potential vorticity anomalies. J. Phys. Oceanogr. 17, 1640-1652.

D'Andrea, F. \& VAUTARD, R. 2000 Reducing systematic errors by empirically correcting model errors. Tellus 52A, 21-41.

Domaradzki, J., LoH, K. \& Yee, P. 2002 Large eddy simulations using the subgrid-scale estimation model and truncated Navier-Stokes dynamics. Theoret. Comput. Fluid Dyn. 15, 421-450.

FArrell, B. \& IoANnou, P. 1995 Stochastic dynamics of the midlatitude atmospheric jet. J. Atmos. Sci. 52, 1642-1656.

FranzKe, C., Majda, A. \& Vanden-Eijnden, E. 2004 Low-order stochastic mode reduction for a realistic barotropic model climate. J. Atmos. Sci. submitted.

GrifFA, A. 1996 Applications of stochastic particle models to oceanographic problems. Stochastic Modelling in Physical Oceanography (ed. R. Adler et al.). Birkhauser, 467 pp.

Griffa, A. \& CASTEllari, S. 1991 Nonlinear general circulation of an ocean model driven by wind with a stochastic component. J. Mar. Res. 49, 53-73.

Haidvogel, D., McWilliams, J. \& Gent, P. 1992 Boundary current separation in a quasigeostrophic, eddy-resolving ocean circulation model. J. Phys. Oceanogr. 22, 882-902.

HAidvogel, D. \& Rhines, P. 1983 Waves and circulation driven by oscillatory winds in an idealized ocean basin. Geophys. Astrophys. Fluid Dyn. 25, 1-63.

Herring, J. \& Kraichnan, R. 1971 Comparison of some approximations for isotropic turbulence. In Statistical Models and Turbulence (ed. M. Rosenblatt \& C. Van Atta), pp. 147-194. Springer.

Holm, D. \& Nadiga, B. 2003 Modeling mesoscale turbulence in the barotropic double gyre circulation. J. Phys. Oceanogr. in press.

Jayne, S., Hogg, N. \& Malanotte-Rizzoli, P. 1996 Recirculation gyres forced by a beta-plane jet. J. Phys. Oceanogr. 26, 492-504.

KaAs, E., Guldberg, A., May, W. \& Deque, M. 1999 Using tendency errors to tune parameterization of unresolved dynamical scale interactions in atmospheric general circulation models. Tellus 51A, 612-629.

Laval, J.-P., Dubrulle, B. \& Nazarenko, S. 2000 Dynamical modeling of sub-grid scales in 2D turbulence. Physica D 142, 231-253.

LaVAL, J.-P., Dubrulle, B. \& McWilliams, J. 2003 Langevin models of turbulence: renormalization group, distant interaction algorithms or rapid distortion theory? Phys. Fluids 15, 1327-1339. 
Majda, A., Timofeyev, I. \& Vanden-Eijnden, E. 1999: Models for stochastic climate prediction. Proc. Natl \& Acad. Sci. 96, 14687-14691.

Majda, A., Timofeyev, I. \& VANDEN-EiJnden, E. 2002 A priori tests of a stochastic mode reduction strategy. Physica D 170, 206-252.

Majda, A., Timofeyev, I. \& Vanden-Eijnden, E. 2003 Systematic strategies for stochastic mode reduction in climate. J. Atmos. Sci. 60, 1705-1722.

Mariano, A., Chin, T. \& Ozgokmen, T. 2003 Stochastic boundary conditions for modeling of coastal flows. Geophys. Res. Lett. in press.

Marshall, J. \& Molteni, F. 1993 Toward a dynamical understanding of planetary-scale flow regimes. J. Atmos. Sci. 50, 1792-1818.

Pedlosky, J. 1987 Geophysical Fluid Dynamics, 2nd edn. Springer, 710 pp.

Press, W., Flannery, B., Teukolsky, S. \& Vetterling, W. 1992 Numerical Recipes. Cambridge University Press, $963 \mathrm{pp}$.

Rhines, P. 1975 Waves and turbulence on a $\beta$-plane. J. Fluid Mech. 69, 417-443.

Rhines, P. 1994: Jets. Chaos 4, 313-339.

Seidov, D. \& Marushkevich, A. 1992 Order and chaos in ocean current dynamics: numerical experiments. Dyn. Atmos. Oceans 16, 405-434.

Siegel, A., Weiss, J., Toomre, J., McWilliams, J., Berloff, P. \& Yavneh, I. 2001: Eddies and vortices in ocean basin dynamics. Geophys. Res. Lett. 28, 3183-3187.

Sura, P. \& Penland, C. 2002 Sensitivity of a double-gyre ocean model to detail of stochastic forcing. Ocean Modelling 4, 327-345.

Treguier, A. \& HuA, B. 1987 Oceanic quasi-geostrophic turbulence forced by stochastic wind fluctuations. J. Phys. Oceanogr. 17, 397-411.

Williams, G. 1978 Planetary circulations: 1. Barotropic representation of jovian and terrestrial turbulence. J. Atmos. Sci. 35, 1399-1426.

Whitaker, J. \& Sardeshmukh, P. 1998 A linear theory of extratropical synoptic eddy statistics. J. Atmos. Sci. 55, 237-258.

ZhANG, Y. \& Held, I. 1999 A linear stochastic model of a GCM's midlatitude storm tracks. J. Atmos. Sci. 56, 3416-3435. 